\title{
La Grande Motte, vingt ans après
}

\author{
par René Bonnefille \\ Laboratoire national d'hydraulique, Chatou \\ et François Rueda
}

Service maritime et de navigation du Languedoc-Roussillon

\section{Introduction}

En 1963 la Mission interministérielle pour l'aménagement du littoral Languedoc-Roussillon, demandait au Laboratoire national d'hydraulique de participer à l'étude sur modèle réduit du port de plaisance de La Grande Motte. Cette participation se concrétisait sous la forme de conseils scientifiques et techniques, auprès du Service spécial d'études maritimes et de génie civil des Ponts et Chaussées, pour la construction et l'exploitation d'un modèle réduit physique à Sète, et les campagnes de mesures en nature utiles pour l'étalonnage de ce modèle.

Le but de l'étude sur modèle réduit concernait uniquement les risques d'ensablement de la passe du port. L'étude a consisté à rechercher une configuration en plan des ouvrages extérieurs du port capable de le protéger contre l'engravement. La question de l'agitation résiduelle dans l'avant-port n'a pas été considérée.

Pour les besoins de l'étalonnage du modèle, un épi témoin a été construit sur le site portuaire en novembre 1964 ; l'évolution des fonds a été suivie jusqu'en août 1965, en parallèle avec des observations de la houle et des expériences de traceurs colorés (LNH, 1966).

Des moyens importants ont donc été mis en œuvre pour concevoir le port de La Grande Motte construit en 1966 et 67 (fig. 1); comme nous allons le prouver ci-après, ces efforts sont récompensés, puisque le port fonctionne bien et ne nécessite aucun dragage d'entretien. Le littoral a évolué comme prévu, à savoir une érosion à l'est du port. Pour pallier cet inconvénient, des travaux d'aménagements complémentaires viennent d'être achevés.

That happened twenty years after the Grande Motte marina building ended.

In 1963, the Grande Motte marina study began on a scale model built at Sète near the site. The main conclusion of the study was that the designed harbour would not suffer heavy difficulties due to littoral sand transports. More than twenty years after the harbour has been built, it appeared usefull to compare the model anticipations and the on site observed events. The study is succesfull as far as sediment movements are concerned; some imperfections on detail behaviour of the scale model are instructive. The coastal protection built on the eastern side of the harbour, with one groin and three breakwaters, is also described in the paper. 


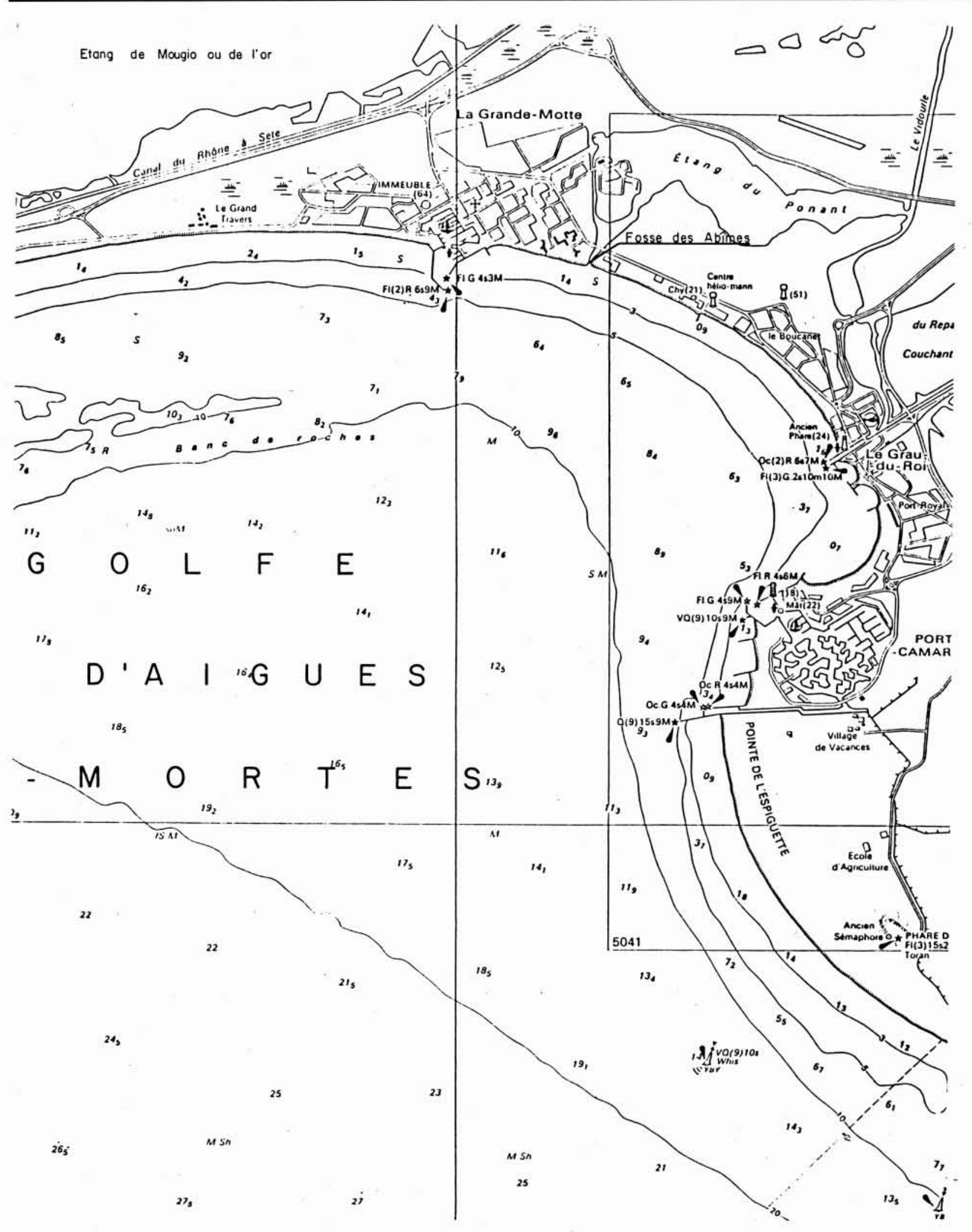

1. Carte du golfe d'Aigues-Mortes

(extrait de la carte marine SHOM n ${ }^{\circ} 7053-1986$ )

Echelle : $1 / 640000$ 


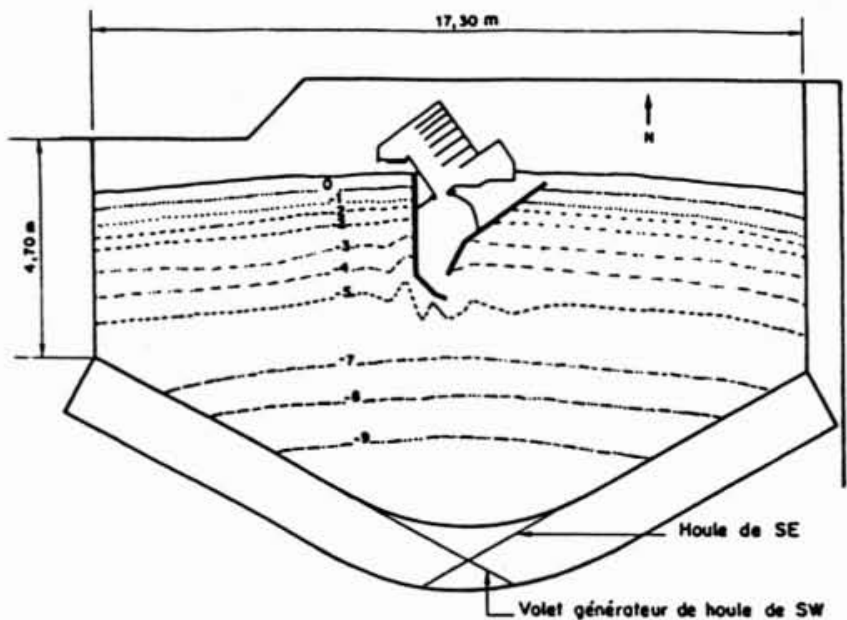

2. Plan schématique du modèle réduit.

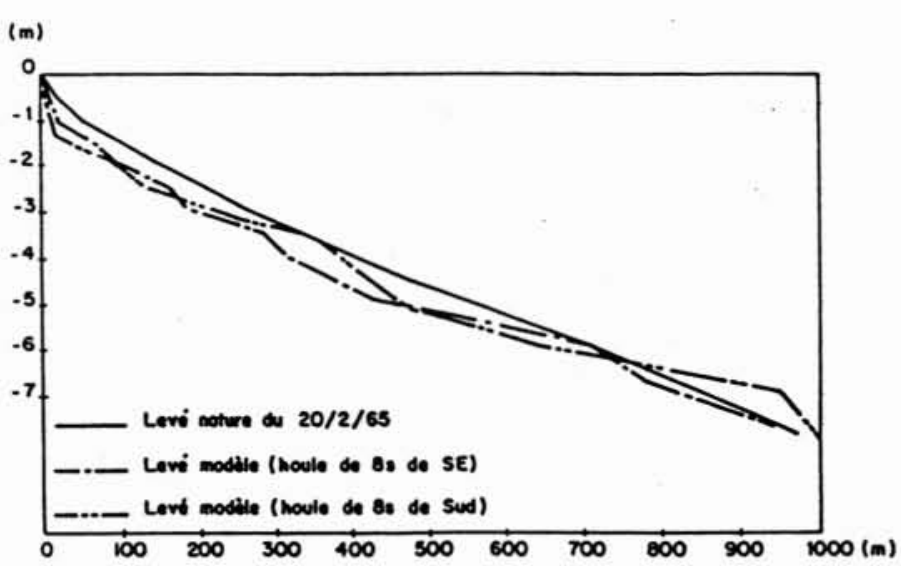

3. Comparaison des profils de plage nature et obtenus sur modèle avec de la houle de hauteur $5 \mathrm{~cm}$ - modèle.

\section{Les principes de la conception du modèle réduit}

Pour des raisons d'économie et de facilité d'exploitation à Sète, le modèle réduit physique utilisait le sable naturel comme matériaux de fonds mobiles. Ses échelles ont été définies de façon à ce que :

- le début d'entraînement des sédiments par la houle ait lieu en des points homologues en nature et modèle ; - la décantation des sédiments en suspension dans la houle se produise suivant des trajectoires homologues en nature et modèle ;

- la réfraction de la houle soit en similitude.

Ces trois conditions conduisent respectivement à ce que (Bonnefille, 1988) :

- le nombre de Reynolds $R^{*}$ construit avec la vitesse de frottement et la taille des grains de sédiment soit le même en nature et modèle;

- le rapport des échelles de similitude de vitesse de chute des sédiments et du fluide soit égal à la distorsion;

- l'échelle de similitude des longueurs d'onde de la houle soit égale à celle de la profondeur.

Désignons par $\hat{d}, \hat{X}, \hat{L}, \hat{H}$ et $\hat{T}$ les échelles de similitude des profondeurs, longueur en plan, longueur d'onde, hauteur et période de la houle. Les conditions ci-dessus se traduisent, lorsqu'on utilise le même sédiment, en nature et en modèle, par :

$$
\hat{L}=\hat{d}=\hat{X}^{4 / 5}, \hat{T}=\hat{X}^{2 / 5}, \hat{H}=\hat{X}^{3 / 5}
$$

Le jeu d'échelles de similitude rondes choisi, satisfaisant presque ces relations, était :

$$
\hat{X}=1 / 200, \hat{d}=1 / 70, \hat{H}=1 / 25, \hat{T}=1 / 8,3
$$

La distorsion était 2,9; en conséquence les cambrures de la houle sur le modèle étaient exagérées dans ce même rapport ; sur le modèle la houle déferlait donc plus au large que dans la nature.

De façon pratique en modèle réduit physique, l'échelle des similitudes des hauteurs de houle n'est pas respectée (car les houles utilisées sur le modèle ne représentent pas les houles nature en similitude chronologique). La hauteur de houle utilisée sur le modèle est en réalité une hauteur "significative" sensée représenter l'action sur les sédiments d'un ensemble de houle provenant d'un secteur particulier pendant une longue période (une saison par exemple). Ceci est obligatoire puisque l'échelle des temps des évolutions sédimentaires est très différente de l'échelle des périodes de houle.

Le modèle réduit (fig. 2), représentait les fonds levés en 1964 , en sable de " taille moyenne " $0,18 \mathrm{~mm}$; son étalonnage a consisté à trouver une schématisation des houles qui l'abordent (de SSE à SW) et qui, en l'absence du port, modèlent une plage semblable à celle observée en nature.

\section{L'étalonnage du modèle réduit}

\subsection{La hauteur de houle à utiliser}

Les données de houle disponibles à l'époque (avril 1964 à juillet 1965) concernaient Palavas; complétées par des plans de réfraction, elles ont permis de définir la houle agissant à La Grande Motte, résumée de la façon suivante, périodes : 6 et $8 \mathrm{~s}$, provenances : $18 \%$ du SW, $2 \%$ du sud, $8 \%$ du SE, $9 \%$ de l'est et $63 \%$ de calme ou de houle très faible à La Grande Motte.

D'abord, fut déterminée, pour les deux périodes 6 et $8 \mathrm{~s}$ et les houles de SW ou SE, la quantité de sable $Q$ à disposer à la limite du modèle, pour que s'établisse un transport littoral constant, en fonction de la hauteur $H$ de houle (par exemple: $Q(1 / \mathrm{s})=0,7 H^{2}(\mathrm{~cm})$ pour $\left.8 \mathrm{~s}\right)$. Ensuite, fut déterminée la hauteur de houle modelant un profil d'équilibre de la plage semblable à celui levé en février 1965, pour les directions de houle sud et SE (SW est équivalent par symétrie). La figure 3 justifie le choix de la valeur $H=$ $5 \mathrm{~cm}$ utilisée sur le modèle pour reproduire un profil de plage d'hiver. Compte tenu de l'échelle théorique des hauteurs $(1 / 25)$, cela signifie que la hauteur de houle significative d'hiver est $1,25 \mathrm{~m}$ (à noter que les hauteurs de houle supérieures à $1,25 \mathrm{~m}$ se manifestent environ $2 \%$ du temps), 


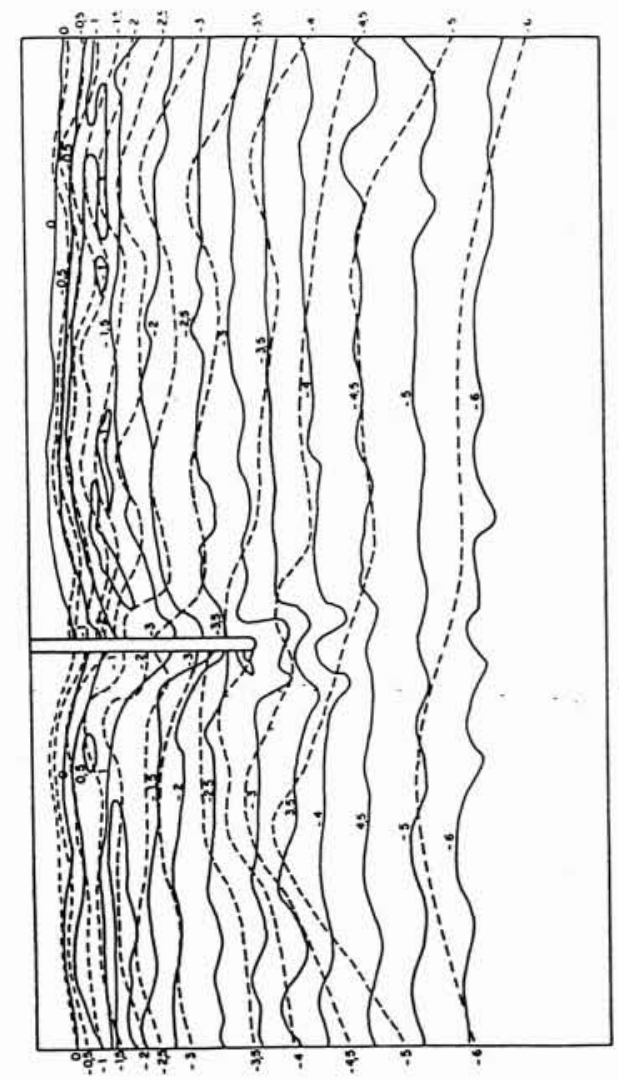

4. Comparaison des évolutions des fonds nature et modèle autour de l'épi expérimental entre le 1/8/64 et le 31/7/65.

Echelle : $1 / 14500$ nature le $(31 / 7 / 65)$ -...- modèle

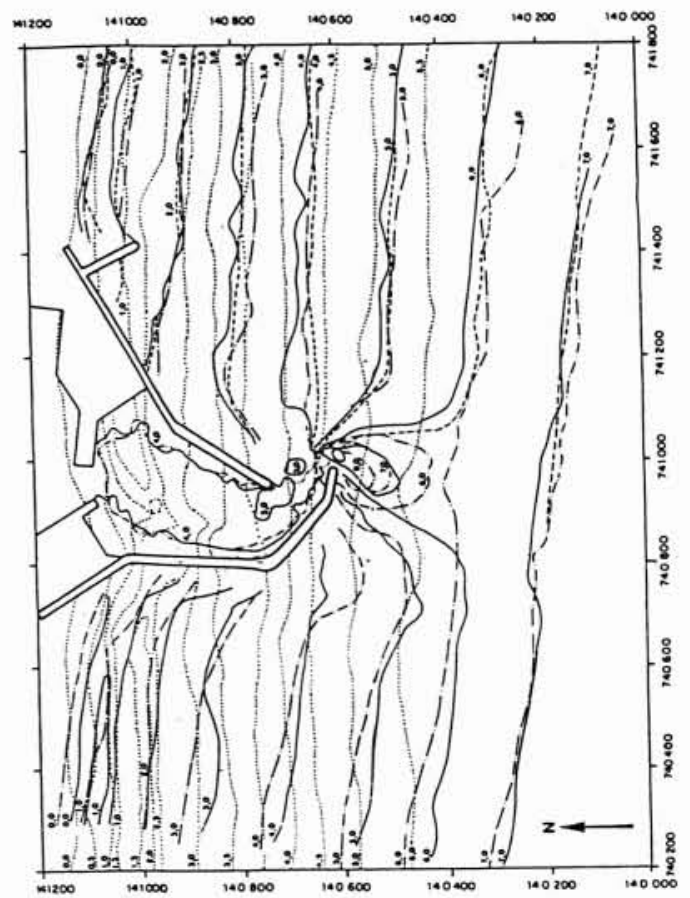

6. Evolution des fonds devant le port de La Grande Motte. 1978 mars 1965 nov. 1982 (avant construction).

Echelle : $1 / 17850$

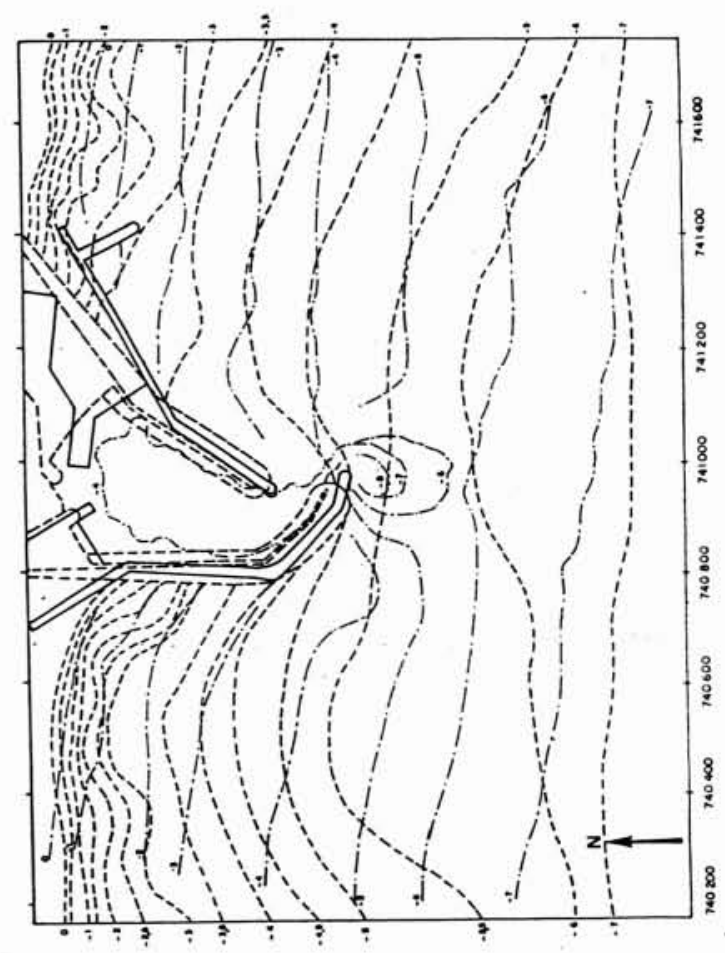

7. Evolution des fonds à partir de mars 1965 _._ 9 ans en nature (mars 1965 à juillet 1974) .... 10 ans en modèle ...- Ouvrages modèle Ouvrages nature

Echelle : $1 / 17850$
5. Evolution des fonds en modèle en 10 ans.
Echelle: $1 / 17850 \quad$ Levé nature du $20 / 3 / 65$ -.-- Levé modèle 10 ans après 


\subsection{L'échelle des temps sédimentologiques}

Un épi expérimental de $370 \mathrm{~m}$ de long, atteignant les profondeurs de $3,5 \mathrm{~m}$, fut construit et l'évolution des fonds voisins suivie ( 5 novembre 1964, 20 février, 20 mars et 31 juillet 1965). La comparaison des levés d'août 1964 et 1965 fait apparaître :

- à l'est, une suite d'engraissement et d'érosion successives ;

- à l'ouest, un déplacement vers le large des lignes bathymétriques, de l'ordre de $60 \mathrm{~m}$ au voisinage de l'épi, indice d'un transport littoral résultant d'ouest en est.

L'échelle du temps sédimentologique fut ensuite estimée, par une série d'essais avec pour but de reproduire au mieux les évolutions des profils de plage entre le 5 novembre 1964 et les 20 février et 20 mars 1965 ( 3,5 et 4,5 mois). Les paramètres utilisés au cours des tâtonnements étaient :

- la hauteur de houle minimale considérée comme agissante : 0,4 et $0,5 \mathrm{~m}$;

- l'échelle des temps d'action des houles agissantes: $1 / 150,1 / 200$ et $1 / 250$.

Les meilleurs résultats concernant les évolutions des profils de plage ont été obtenus en prenant en compte les houles de hauteurs supérieures à $0,4 \mathrm{~m}$ et l'échelle des temps d'action $1 / 200$. Compte tenu de la réfraction entre le lieu d'observation de la houle (Palavas) et La Grande Motte, et la durée des calmes (c'est-à-dire les hauteurs de houle inférieures au seuil), l'échelle des temps sédimentologiques a été estimée à $1 / 800$.

Enfin, fut mise au point la série chronologique des houles (direction, hauteur et période) sensée représenter une année d'action de la houle. La série fut mise au point par tâtonnement (en particulier les hauteurs des plus fortes vagues ont dû être réduites, car elles déferlaient sur le modèle distordu au lieu d'être actives comme en nature) en reproduisant les évolutions des fonds autour de l'épi entre août 1964 et juillet 1965 (la figure 4 montre les relevés des fonds nature et modèle, après un an d'évolution nature et modèle). La reproduction a été jugée qualitativement satisfaisante : elle est bonne du point de vue profil de plages. Il apparaît toutefois une différence de profondeur systématique de $50 \mathrm{~cm}$ à $1 \mathrm{~m}$ entre le modèle et la nature (le profil modèle est plus creusé que le profil nature) due au fait que, compte tenu de la distorsion, la barre de déferlement nature, au voisinage des profondeurs de $1,5 \mathrm{~m}$, n'est pas reproduite sur le modèle.

Il convient de signaler que des fosses importantes se sont creusées au pied du brise-lames ouest ; ce phénomène jugé comme "parasite " a été supprimé en réduisant le coefficient de réflexion des ouvrages exagéré par la distorsion du modèle.

\section{L'étude du projet de port}

\subsection{Le comportement en modèle}

L'implantation du projet de port sur les fonds de juil- let1965 a demandé, comme précaution spéciale, la mise au point d'un profil de brise-lames convenable du point de vue réflexion de la houle, de façon à éviter la formation trop accentuée de fosses devant les ouvrages (le profil naturel a la pente 2/1). La figure 5 donne le résultat des évolutions des fonds après 10 ans. On note:

- le maintien de grandes profondeurs (4 à $5 \mathrm{~m}$ ) sur la face SW du brise-lames de l'ouest, ce qui a un effet bénéfique empêchant le contournement de l'ouvrage par le transport littoral ;

— des dépôts sur la face NE de ce même brise-lames, dans la passe d'entrée, mais ces dépôts ne sont pas très importants ;

- en 10 ans, le littoral a progressé à l'ouest (70 m environ) et régressé à l'est (40 m environ); la progression est locale, tandis que la régression affecte toute la longueur de plage.

Les conclusions de l'étude de 1966 étaient donc:

- la forme du port est satisfaisante, pour la zone de La Grande Motte où le transport littoral est faible ;

- la pente $2 / 1 \mathrm{du}$ brise-lames est un choix judicieux, car il permet le creusement de fosses de clapotis encore acceptables ;

- la ligne de rivage évoluera, mais assez lentement pour permettre la mise en place des ouvrages de préservation de la côte.

\subsection{Le comportement en nature}

Le port a assez bien tenu les promesses de l'étude :

- il est sûr du point de vue agitation, ce qui avait été prévu par les études préliminaires sur plan ;

- l'ensablement de l'avant-port est nul (sur le modèle aussi) ;

— le littoral a avancé à l'ouest; il a reculé à l'est, en causant quelques dégâts; il en a résulté la construction d'ouvrages de protection du littoral décrits plus loin.

Grâce aux nombreux levés bathymétriques effectués de 1965 à 1982, on a pu reconstituer les évolutions des fonds marins pendant une vingtaine d'années (fig. 6). La figure 7 permet de comparer les évolutions des fonds sur 9 ans en nature (mars 1965 à juillet 1974) et sur 10 ans en modèle. Les lignes bathymétriques sont celles de la figure 5 pour le modèle et celles de la figure 6 pour la nature. La comparaison des deux ensembles de lignes bathymétriques, avec des ouvrages portuaires un peu différents, est basée sur la coïncidence des levés du 20 mars 1965 en nature et en modèle. Le bon accord modèle-nature concerne l'avancée du rivage à l'ouest du port. A l'est du port la construction de l'épi en nature a empêché le recul des fonds, qui par exemple atteint $150 \mathrm{~m}$ à $200 \mathrm{~m}$ aux profondeurs $2 \mathrm{~m}$ à $4 \mathrm{~m}$ sur le modèle (fig. 5). La figure 6 montre qu'au contraire les lignes bathymétriques -3 et $-4 \mathrm{~m}$ ont avancé de $75 \mathrm{~m}$ environ vers le large.

Les érosions importantes qui se sont manifestées au musoir du brise-lames de l'ouest (creusement d'une fosse de $4 \mathrm{~m}$ en 1974) ne sont pas apparues sur le modèle ; il faut noter que ces érosions se sont résorbées en nature en partie 


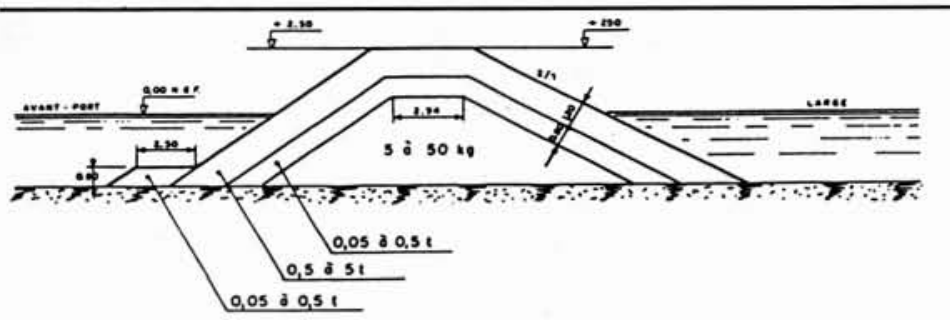

Profit initial

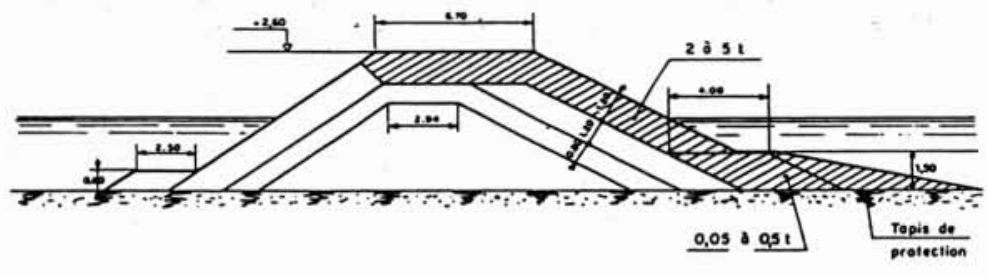

Profit renforcé modifié

8. La modification du profil de brise-lames.

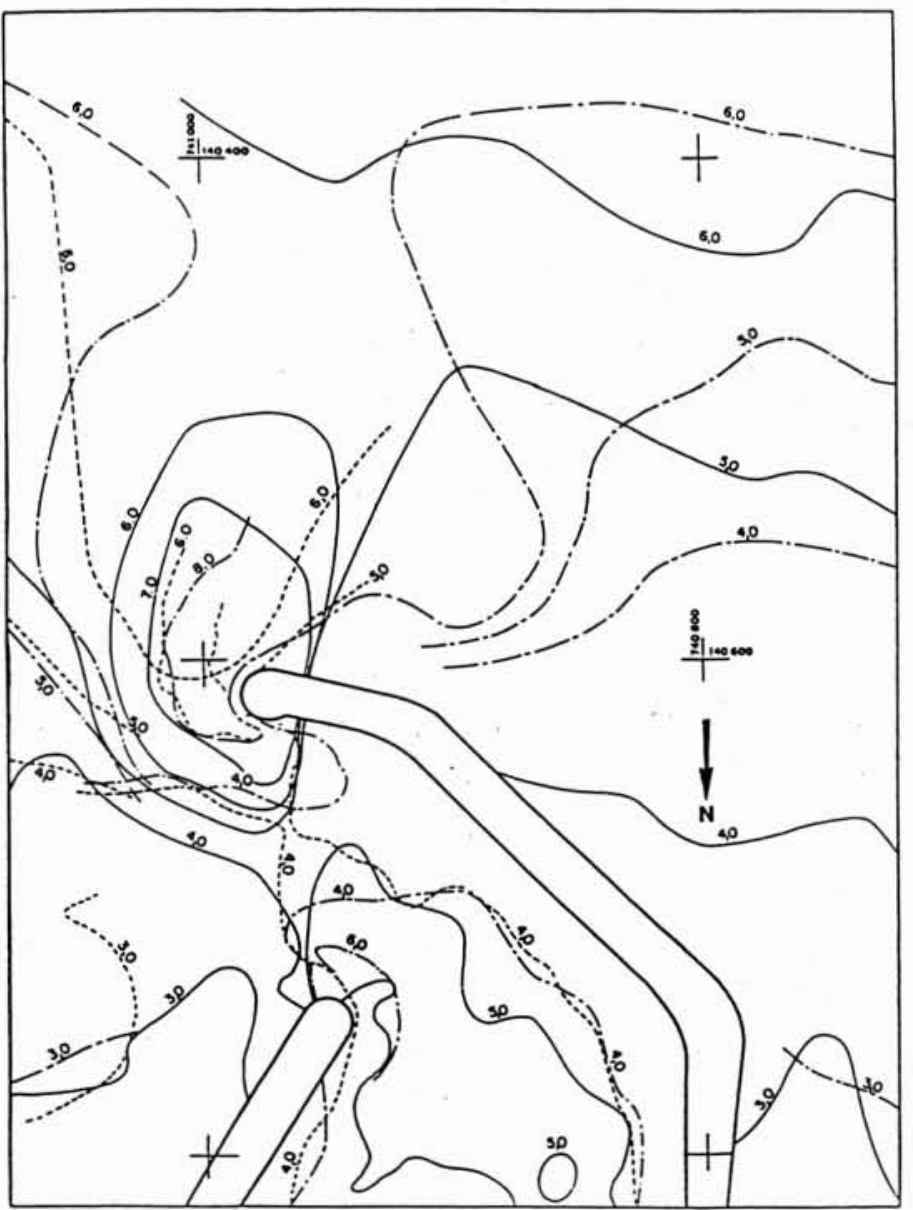

9. Evolution des fonds autour de la passe du port de La Grande Motte.

$\frac{1982}{192}$ mars $1970 \quad$-. - - juin $1976 \quad$-.-. mars

Echelle : $1 / 4000$ en 1978 (fig. 6). Les dépôts derrière le brise-lames ouest observés en modèle, ne se sont pas produits en nature ; au contraire en 1978 il est apparu des érosions dans la passe d'entrée de l'avant-port (fig. 7).

La conclusion est que les évolutions des fonds marins n'ont été prédits que globalement par le modèle, mais ceci avec succès dans leur partie essentielle, à savoir qu'il n'y avait pas risque de contournement du musoir du briselames ouest par le transport littoral.

\subsection{Les enseignements à tirer du point de vue de la simi- litude}

Du point de vue " utilisation des modèles réduits à fonds mobiles ", parfois tant controversés, l'étude est un franc succès. Elle a même révélé des erreurs à éviter, citées plus loin. Rappelons :

- les données de la similitude

- échelles en plan 1/200, profondeur $1 / 70$, hauteur de houle $1 / 25$,

- fonds mobiles en sable de la nature,

- échelles des temps action de la houle $1 / 200$, évolution à long terme $1 / 800$.

- les bases de l'étalonnage

- la reproduction des profils de plage et de leur évolution autour d'un épi expérimental pendant quelques mois, a permis de déterminer l'échelle des temps d'action de la houle $(1 / 200)$; compte tenu du dosage des hauteurs de houle à faire agir sur le modèle, et des effets de la réfraction, cette mise au point relègue l'utilisation de l'échelle des hauteurs de houle $(1 / 25)$ à avoir un rôle secondaire, ou plutôt seulement indicatif pour la préparation des premières séries chronologiques de houle à utiliser;

- la reproduction de l'évolution des fonds autour de l'épi expérimental pendant un an, a permis de mettre au point la série chronologique de la houle à utiliser et d'en déduire l'échelle de temps des évolutions de fonds $(1 / 800)$;

- la reproduction correcte de la réflexion de la houle sur les ouvrages en adaptant leur structure pour compenser les effets de la distorsion.

\subsection{Les enseignements à tirer du point de vue de la concep- tion des ouvrages}

Le profil initial des brise-lames du port de La Grande Motte a été déterminé par application d'une formule intermédiaire entre celles de Beaudevin et d'Hudson. La stabilité de ce profil avec talus à $2 / 1$ a été vérifiée par profondeur de $5 \mathrm{~m}$ par Sogreah. Le profil s'est révélé stable sur fonds non affouillables; en revanche la carapace a été désorganisée du fait des affouillements en pied de l'ouvrage. Le profil a donc été modifié par renforcement du couronnement et de la carapace appuyée sur une butée de pied à talus à $5 / 1$ (fig. 8 ). Ce profil a ensuite donné satisfaction.

Il est utile d'ajouter que ces affouillements constatés près du musoir, dus aux courants de houle et au clapotis, étaient 
prévisibles au niveau de l'étude sur plan; mais ils ne pouvaient pas se manifester dans l'étude de la stabilité du profil en canal à houle à fonds non affouillables. Rappelons qu'ils étaient apparus sur le modèle sédimentologique, mais avaient été considérés comme des parasites liés à la distorsion des échelles du modèle.

Du point de vue de la conception des ouvrages, on peut retenir de cette étude, l'importance du profil des carapaces des brise-lames. Les profils trop réfléchissants sont, dans les mers sans marée, la cause de creusements de fosses importantes devant les ouvrages. Le clapotis crée en outre des remises en suspension des sédiments charriés par le transport littoral dominant; ces sédiments se déposent devant la passe où l'effet du clapotis est réduit; ils sont repris ensuite par le clapotis dû à des houles de directions différentes; ces houles génèrent un transport littoral opposé qui, même faible, introduit ces sédiments dans la passe oủ ils restent, faute de courants de marée de vidange du port pour les évacuer.

Ces remarques ne condamnent pas l'utilisation des ouvrages à profils raides, dans la mesure où ils sont substantiellement économiques. Toutefois elles montrent que les ouvrages à profil raide peuvent causer des évolutions des fonds qu'il vaut mieux prévoir et en estimer les inconvénients pour juger de la faisabilité d'un projet.

\section{Vingt ans d'histoire du port de La Grande Motte}

Grâce aux observations et mesures du Service maritime et de navigation du Languedoc-Roussillon de Sète et de la capitainerie du port, on peut tirer d'autres enseignements régionaux utiles aux aménagements.

\subsection{Les tempêtes}

Depuis 1967, la tempête la plus importante qu'a subie le port est celle de SE des 5-6 décembre 1982, tempête de période de retour centennale (elle correspond d'ailleurs à celle de 1890). La surcote qui accompagnait la tempête était également exceptionnelle: certains quais ont été recouverts de $30 \mathrm{~cm}$ d'eau à La Grande Motte ; le niveau de la mer a donc atteint la cote $+1,6$ m NGF $(+1,35$ à Sète en 1890). La rareté du phénomène justifie de ne pas en tenir compte pour établir la cote d'arase des quais; toutefois une telle surcote est à considérer pour concevoir les brise-lames du Golfe du Lion.

Les seiches qui prennent naissance dans le port ne sont pas trop gênantes, à part les courants alternatifs dans la passe entre l'avant-port et les bassins du port.

Lors de l'événement de la nuit du 5-6 août 1985 (oscillation du niveau de la mer de 20 à $30 \mathrm{~s}$ pendant $3 \mathrm{~h}$ environ), surcote de $0,8 \mathrm{~m}$ et décote de $1 \mathrm{~m}$ à La Grande Motte), les dégâts aux embarcations ont été importants du fait de la décote, les bateaux s'étant coincés sous les quais.

\subsection{L'évolution des fonds marins}

La figure 6 concernant les abords immédiats du port et la figure 9 concernant l'intérieur de l'avant-port représentent les évolutions des fonds marins pendant une vingtaine d'années.

Le fait important est le creusement d'une fosse au droit du musoir du brise-lames dans la passe. Cette fosse a atteint la cote maximale de $-8 \mathrm{~m}$ en 1976 (soit $3 \mathrm{~m}$ sous le niveau des fonds voisins); elle s'est ensuite en partie comblée en 1982. Rappelons encore qu'un phénomène semblable s'était manifesté sur le modèle, dû aux courants de houle; il a été à tort considéré comme phénomène parasite et donc supprimé pour la poursuite de l'étude. Si le modèle n'avait pas été distordu, de tels phénomènes s'étant manifestés, ils auraient été pris obligatoirement en compte.

A l'est du port, les profondeurs n'ont pratiquement pas varié de 1965 à 1982 (fig. 6), tout au moins dans la zone proche du port; en revanche le modèle avait prévu une érosion de l'ordre de $1 \mathrm{~m}$ au niveau de la passe du port et de $1,5 \mathrm{~m}$ près du rivage (fig. 7 ). Cette érosion a pu être évitée localement grâce à la construction du tenon enraciné sur le brise-lames est. Mais le littoral a régressé plus à l'est, conduisant à la construction en 1987 de brise-lames parallèles au rivage pour protéger le littoral contre l'érosion. Rappelons que le modèle n'avait pas une emprise suffisante pour reproduire ce phénomène.

\section{La protection du littoral à l'est du port}

\subsection{L'état des lieux}

A l'est de La Grande Motte, la plage se développe sur $1 \mathrm{~km}$ de longueur jusqu'à la passe des Abîmes, ancien débouché en mer d'une rivière côtière, le Vidourle (fig. 1). L'amaigrissement de cette plage extrêmement fréquentée par la population locale et estivale mettait en péril l'activité balnéaire de la station touristique, si bien que les plagistes (location de pédalos et parasols) furent invités à replier leurs installations.

Des apports de sable ( $5000 \mathrm{~m}^{3}$ environ) effectués chaque année au mois de mai disparaissaient régulièrement l'hiver venu. La moindre tempête ruinait un peu plus les ouvrages de haut de plage (escaliers, murets, postes de secours). De 1981 à 1985 , la plage a perdu 10 à $20 \mathrm{~m}$ de largeur. Les évaluations du modèle réduit, que l'on avait un peu oublié, se confirmaient donc. En 1983, la commune de La Grande Motte chargeait le Service maritime et de navigation du Languedoc-Roussillon d'établir un avant-projet technique des travaux à réaliser.

Cette étude s'intégrait parfaitement au Schéma directeur de protection des départements de l'Hérault et du Gard (105 km de façade maritime), ce schéma définissant les objectifs, les priorités et les techniques à mettre en œuvre dans le cadre d'une réflexion globale de programmation et de défense du littoral des deux départements. 


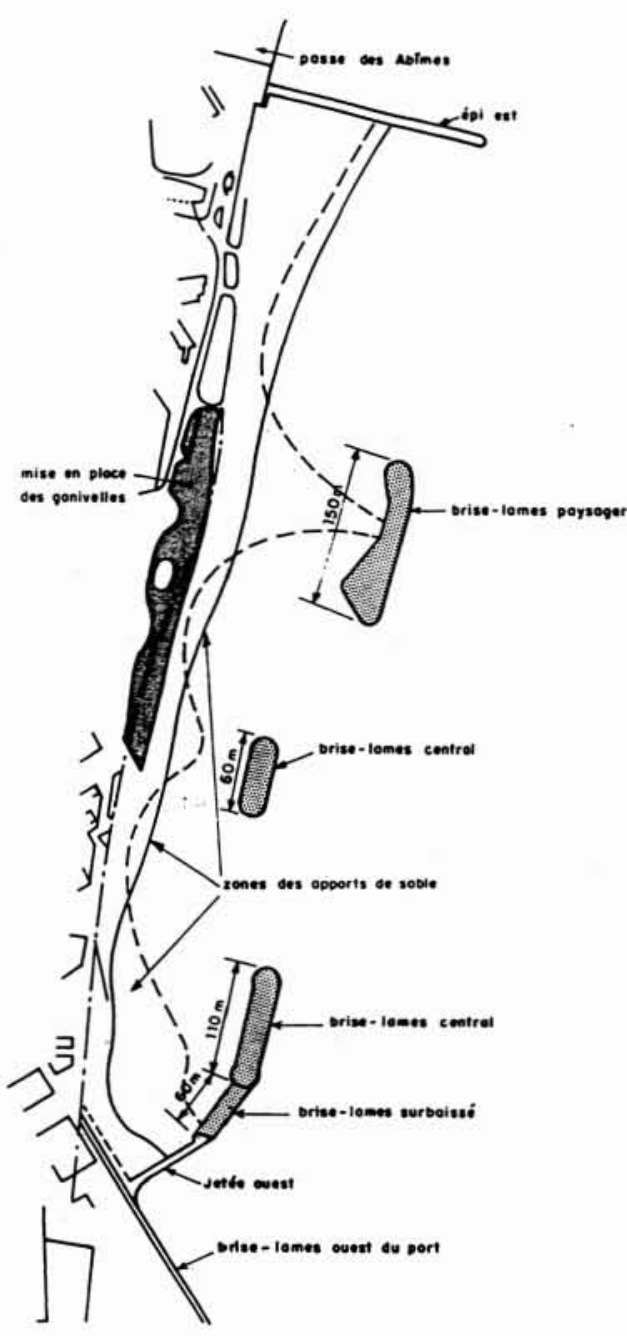

10. Le projet de protection du littoral à l'est du port de La Grande Motte.

_ laisse avant travaux (mars 1987) laisse après travaux (juin 1988)

Echelle : $1 / 10000$

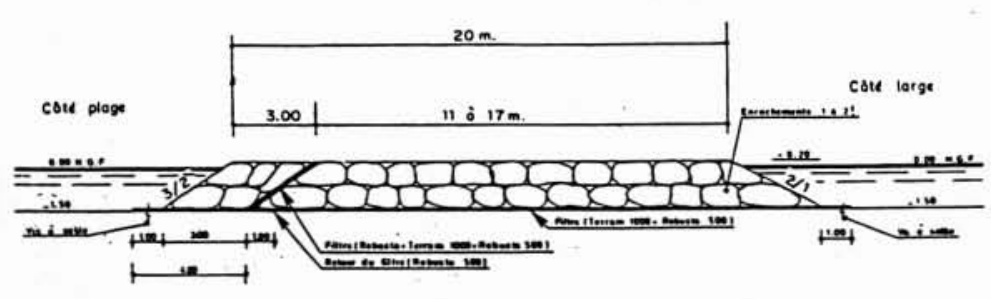

11. Profil en travers du brise-lames surbaissé - Cotes en mètres. Echelle : $1 / 400$

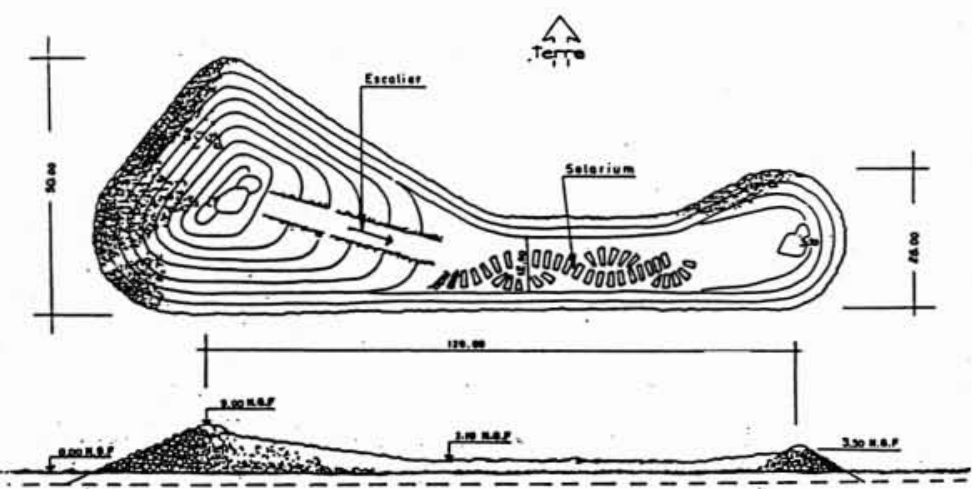

12. Le brise-lames paysager - Cotes en mètres. Echelle : 1/2000
Précédé d'une étude diagnostic, destinée à apprécier les facteurs responsables du comportement du littoral et à en déceler les évolutions futures, ce schéma fut donc appliqué. Ensuite des relevés systématiques de profils à terre et en mer ont permis de fournir pour l'avenir des renseignements indispensables et d'ajuster les moyens de protection aux évolutions observées.

\subsection{L'étude de la protection de la plage}

L'étude a été menée avec l'aide de la Sogreah (sédimentologie) et du BCEOM (impact sur l'environnement). Un diagnostic a révélé les causes de l'érosion :

- absence d'alimentation en sédiments aussi bien côté mer que côté terre,

- présence du port de plaisance qui bloque la majeure partie des transports littoraux,

- attaque frontale des houles,

- arrière-plage constituée d'un front de mer urbanisé.

L'étude a précisé celle de 1965 en montrant que les houles de provenance SSE à SSW, de période $8 \mathrm{~s}$, sont porteuses du maximum du flux d'énergie et engendrent un transit littoral résultant dirigé vers l'est ; de plus la composante frontale de l'attaque des houles n'est pas négligeable.

Fort de cette analyse, confortée par des levés topographiques et bathymétriques réguliers, des analyses granulométriques des sables en place (en mer et à terre) et des photos aériennes, l'étude a conclu à la nécessité de bloquer la plage aux limites, de façon à constituer une unité à l'intérieur de laquelle le débattement du trait de côte, dû aux variations du régime de houles, serait atténué et oủ la largeur de la plage serait suffisamment augmentée pour offrir une protection naturelle contre les jets de rive.

\subsection{L'épi est (première tranche de travaux)}

Afin de valider les résultats de l'étude, un premier épi d'une longueur de $230 \mathrm{~m}$, fut implanté en 1984 (fig. 10), par des fonds à $-2,50 \mathrm{~m}$ à l'extrémité est de la plage. L'épi a parfaitement joué son rôle d'ouvrage d'accumulation et assuré de manière remarquable la protection du secteur correspondant à sa zone d'influence. A l'enracinement de l'épi, la plage a avancé de $90 \mathrm{~m}$. En revanche, l'érosion à l'ouest (vers le port) et que l'on pouvait craindre s'est poursuivie, la plage ayant tendance à pivoter autour d'un axe central.

\subsection{Les brise-lames (deuxième phase de travaux)}

Les observations sur le comportement du littoral après réalisation du premier ouvrage ont permis d'apprécier l'importance des ouvrages de deuxième tranche. A ce stade, trois solutions ont été envisagées, dont les différences tenaient à l'alternative épis ou brise-lames, respectant toutes l'idée d'un cloisonnement de la plage en plages élémentaires.

Les brise-lames ont finalement été retenus parce qu'ils 
encombraient moins la plage, permettaient un engraissement localisé (formation de tombolos) et assuraient une meilleure protection du front de mer contre l'attaque frontale des houles et la surélévation du niveau de la mer lors des tempêtes (fig. 10). Ce dispositif fut complété par un apport de sable indispensable et exécuté pendant la phase travaux, les caractéristiques de ce sable devant satisfaire aux conditions suivantes relatives au diamètre des grains : $\mathrm{D}_{50}$ supérieur à $0,2 \mathrm{~mm}$ et $\mathrm{D}_{84} / \mathrm{D}_{16}$ inférieur à 4 .

\subsubsection{Le brise-lames surbaissé}

L'étude d'impact montrait que les trois ouvrages parallèles à la côte auraient un impact très fort sur la station de La Grande Motte, marquée par la qualité architecturale de ses pyramides. L'idée de faire disparaître les brise-lames sous l'eau conduisait à envisager la possibilité de réaliser des digues immergées ou tout au moins arasées au niveau moyen de la mer.

Devant le manque de connaissances dans ce domaine, le coût probable de tels ouvrages et le doute quant à leur efficacité sur une plage fortement réduite et agressée, l'idée fut rejetée pour les deux ouvrages les plus à l'est, prévus arasés à des cotes allant de $+1,8 \mathrm{~m}$ à $2,1 \mathrm{~m}$. En revanche, le brise-lames ouest se prêtait à une expérience de ce type car il pouvait apporter une amélioration de la qualité des eaux de la plage. En effet, l'ouvrage, par sa longueur $(250 \mathrm{~m})$, était porteur de risque de confinement des eaux, rétention d'objets flottants et développement algal. Par précaution, c'est une "fenêtre " surbaissée de $60 \mathrm{~m}$ qui a été réalisée suivant le profil en travers de la figure 11. Il présente les particularités suivantes:

- absence de noyau: la digue est simplement constituée d'enrochements de $2^{\mathrm{e}}$ catégorie ( 1 à 2 tonnes) ;

- pose des enrochements sur un filtre en géotextile pour éviter l'ensouillement :

- étanchéité aux sables qui pouvaient fuir de la plage vers la mer ; cette étanchéité est réalisée au moyen de filtres en géotextile.

\subsubsection{Le brise-lames paysager}

La conception du brise-lames le plus à l'ouest était diamétralement opposée. Alors que les efforts allaient vers des ouvrages les plus bas possibles sur l'eau, Jean Balladur, architecte en chef de La Grande Motte, à qui avait été soumis le plan de protection de la plage, proposa au contraire d'en faire un signal volontaire dans le paysage. Imaginé comme deux promontoires reliés par une partie horizontale, l'ouvrage émerge à $+9,00 \mathrm{~m}$ et $+3,50 \mathrm{~m}$ (fig. 12).

Une adaptation technique du dessin se révéla nécessaire, mais l'esprit d'un aménagement paysager fut totalement respecté. Un escalier en béton permettant d'accéder au promontoire le plus élevé a été réalisé et une partie des blocs en enrochements de la partie centrale ont été remplacés par des éléments en béton de même poids pour constituer des solariums.

D'une longueur de $120 \mathrm{~m}$ dans l'axe des musoirs, fondé à la cote $-2 \mathrm{~m}$, distant de $140 \mathrm{~m}$ par rapport au rivage d'origine, le brise-lames paysager a coûté $85 \%$ de plus qu'un ouvrage classique.

\subsection{Les apports de sable}

En présence d'un brise-lames, la diffraction des houles autour des musoirs crée un tombolo qui se constitue au détriment du stock de sable disponible dans les zones voisines. Il faut donc impérativement procéder à des apports de sable provenant de l'extérieur du site sous peine de voir ces zones s'appauvrir en matériaux et s'éroder plus activement qu'en l'absence d'ouvrages de protection. La difficulté de ces apports de sable réside dans le fait qu'il est nécessaire de trouver un sable extérieur dont la granulométrie est supérieure à celle du sable en place. En Languedoc, cette difficulté devient la plupart du temps une impossibilité.

Aussi, lorsque l'on dispose de sable extérieur de qualité inférieure qui risque de ne pas tenir sur la plage, est-il préférable de le mettre en place dès le début du chantier sur les côtés extérieurs du brise-lames, de façon à ce qu'il soit repris par les houles et constitue l'âme du tombolo, le sable de la plage venant naturellement s'étaler en couverture dans une deuxième phase. Le volume de sable à disposer doit en tout en état de cause être égal au volume du tombolo probable. L'intérêt de cette manœuvre est de saturer immédiatement le brise-lames, ce qui permet de réduire les effets secondaires et d'obtenir dès la fin des travaux le profil définitif de la plage.

Dans le cas de La Grande Motte, le manque de sable de bonne qualité a conduit à utiliser deux sites d'emprunt : la pointe de l'Espiguette et le port de plaisance. La flèche sableuse de l'Espiguette sur laquelle a été implanté le port de plaisance de Port Camargue avance régulièrement de $10 \mathrm{~m}$ par an (figure 1). Cette zone en fort engraissement constitue une réserve naturelle dans laquelle des volumes de sable conséquents peuvent être prélevés sans détruire l'équilibre des plages environnantes.

Le transport par voie routière s'avérant trop onéreux, l'apport de sable a été effectué à l'aide d'engins maritimes. Deux dragues étaient en service. La première aspirait le sable, le déposait dans son puits et se déplaçait pour claper le matériau devant la plage à protéger par des fonds de - $5 \mathrm{~m}$ environ. La seconde qui stationnait sur cette zone de dépôt refoulait sur la plage à l'aide d'une conduite flottante. $17300 \mathrm{~m}^{3}$ ont ainsi été déposés sur la plage.

Le port de plaisance a servi de deuxième zone d'emprunt, la drague stationnaire refoulant directement sur la plage. Le sable extrait de l'avant-port était remplacé, au fur et à mesure de l'exécution du chantier, par du sable prélevé à l'Espiguette et transporté à La Grande Motte par la drague aspiratrice en marche. Cette deuxième opération a permis de mettre en place $39900 \mathrm{~m}^{3}$ de sable. L'avantage de cette manipulation était principalement de mettre à l'abri des houles la drague stationnaire qui pouvait ainsi travailler en continu, mais également de disposer d'un stock de sable contenu dans les limites physiques des digues de l'avant-port. 


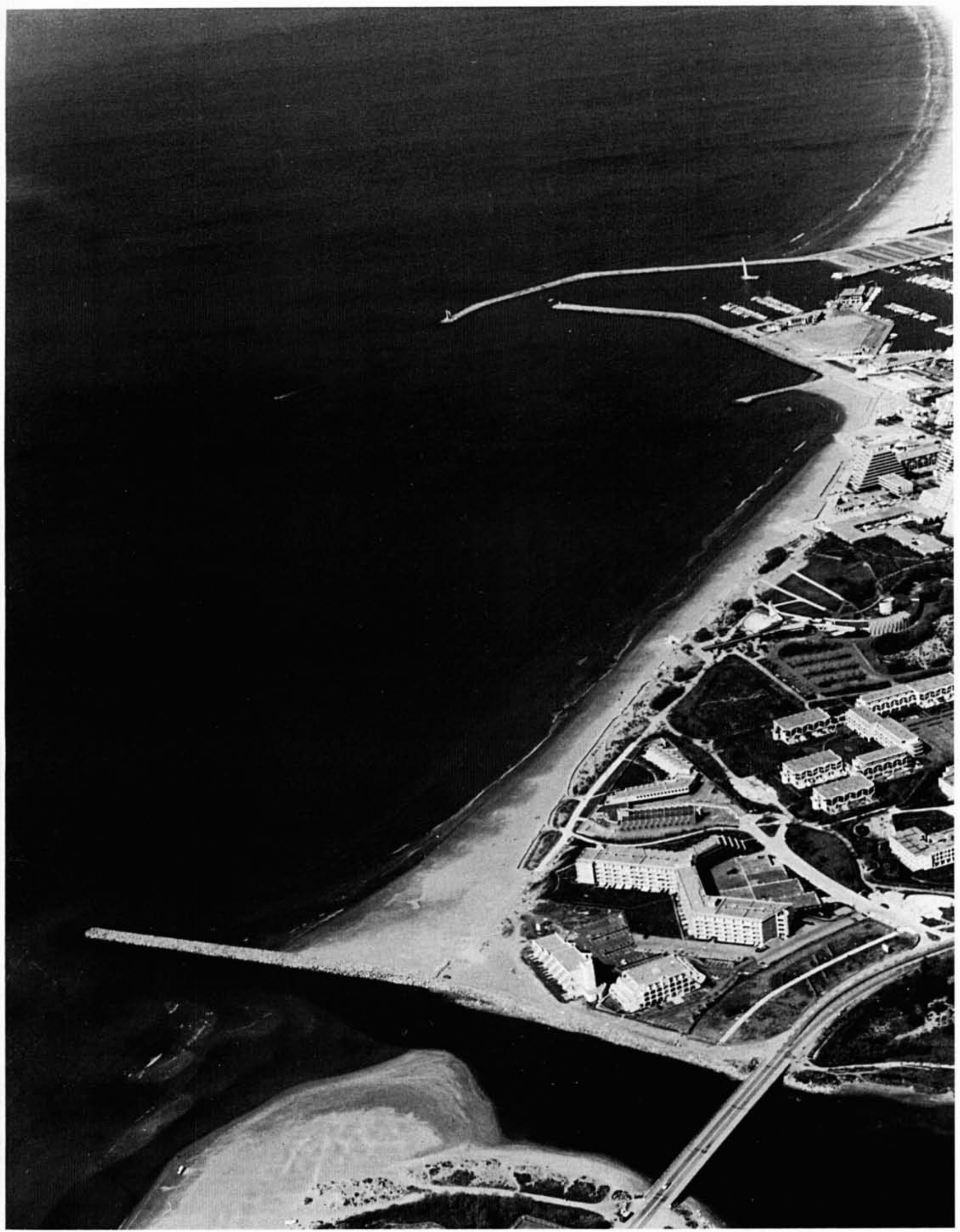



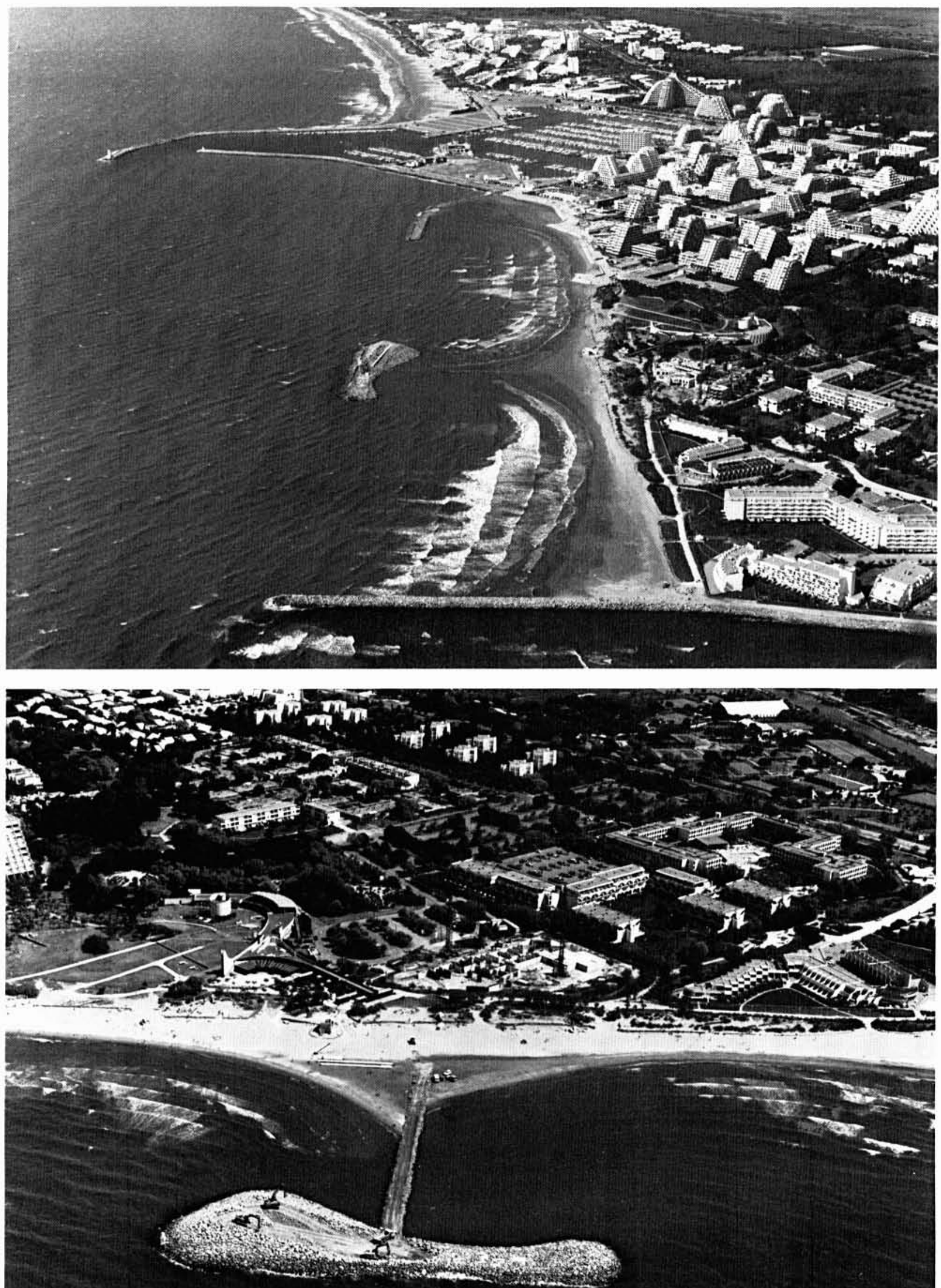


\subsection{Les dunes}

L'aménagement de La Grande Motte en 1966 s'est traduit par la destruction des dunes littorales sur lesquelles s'est édifiée la station nouvelle. Or les dunes bordières constituent un élément essentiel de l'équilibre dynamique de la plage. Bien qu'elles ne puissent répondre seules au problème de l'érosion, leur préservation et leur restauration doivent accompagner tout projet de protection du littoral.

Dans le cadre du programme de travaux une série de trois lignes de ganivelles a donc été installée. Les deux premières sont parallèles à la plage et espacées de $5 \mathrm{~m}$ environ. La troisième met en défense la dune contre le piétinement. Complété d'un clayonnage tous les $10 \mathrm{~m}$ et de six passages publics, les ganivelles représentent un développé de $1520 \mathrm{~m}$ pour un linéaire de $350 \mathrm{~m}$ de plage.

Nota : Les ganivelles sont des barrières en piquets de châtaignier refendu, liées par du fil de fer galvanisé, de hauteur 1,2 à $1,5 \mathrm{~m}$, et assez écartées pour permettre une perméabilité au vent.

\section{Conclusion}

Indépendamment de sa réussite économique, le port de plaisance de La Grande Motte est une réussite technique en ce sens qu'il ne pose pas de gros problèmes du point de vue entretien, et qu'il assure sa fonction d'abri. Son seul inconvénient, les érosions à l'est, peut être maîtrisé par des ouvrages peu importants.

On peut conclure que le modèle réduit, même construit avec des fonds en sable, a rempli son rôle, en laissant "espérer", ce qui s'est produit, une bonne stabilité des fonds marins. Comme il a amorcé l'érosion à l'est, en l'amplifiant même, on peut dire qu'il a rempli son rôle "d'indicateur». L'amorce de ces érosions, et la tendance des dépôts à l'ouest du port, montrent que le modèle aurait été capable de faire apparaître des évolutions des fonds importantes, si elles avaient dû se produire en un site moins stable que celui de La Grande Motte. Ces considérations effacent les regrets des concepteurs de n'avoir pu représenter sur le modèle réduit une longueur de rivage plus grande, limitée à l'époque par les dimensions du hangar disponible au port de Sète.

Commencée en 1983 par l'étude et terminée en 1988 par la mise en place des ganivelles, la protection de la plage de
La Grande Motte a coûté 8400000 F/TTC 1988. La maîtrise d'ouvrage a été assurée par le Département de l'Hérault qui a financé $20 \%$ du montant des travaux, le complément provenant de la Commune, l'Etat ( $1^{\text {re }}$ phase), et la région Languedoc-Roussillon ( $2^{\mathrm{e}}$ phase).

Rappelons que cette opération comportait plusieurs points remarquables :

- étude fine du littoral,

- réalisation d'un ouvrage en $1^{\text {re }}$ phase,

- suivi du comportement de la plage,

- proposition des ouvrages de $2^{\mathrm{e}}$ tranche,

- prise en compte de l'environnement: brise-lames paysager,

- utilisation des géotextiles,

- expérience d'un ouvrage surbaissé,

- apport de sable conséquent,

— restauration du milieu dunaire.

Les ouvrages n'ont encore subi qu'un seul hiver et les conclusions hâtives doivent être évitées. Cependant l'on peut penser, au vu du comportement de la plage pendant quelques tempêtes, que l'ensemble des travaux menés pour la protection de ce littoral sont d'une efficacité réelle. L'on se plaît pourtant à imaginer ce qu'aurait pu être cette plage si les indications fournies par le modèle réduit avaient été suivies d'effets. Puisque le modèle a été capable d'annoncer les évolutions importantes qui se sont produites, il est regrettable que :

- les aménageurs n'aient à l'époque imaginé un front de mer moins urbanisé, moins dur, ou tout au moins plus éloigné du rivage ;

- la protection de la plage n'ait pu être menée dès l'origine ou presque du port de La Grande Motte, ce qui nous aurait valu aujourd'hui des ouvrages probablement moins conséquents.

En matière d'aménagement du littoral, l'érosion irréversible des plages doit être un élément à prendre en compte, et les réflexions qu'elle suppose doivent accompagner tout projet de construction proche du rivage. C'est ainsi qu'il serait souhaitable de parler d'aménagement du littoral (l'érosion est une contrainte) plutôt que de protection du littoral (on attend le seuil d'irréversibilité pour agir). Car protéger, défendre, préserver, c'est déjà admettre que la lutte avec les éléments naturels est engagée... ou presque perdue.

\section{Bibliographie}

L.N.H. (1966). - Etude sur modèle réduit du port de plaisance de La Grande Motte, T 474 DHM, Laboratoire national d'hydraulique, 6, quai Watier, 78400 Chatou, février 1966.

R. Bonnefille. - La Grande Motte, vingt ans après, HE40/88.08A, Laboratoire national d'hydraulique, 6, quai Watier, 78400 Chatou, mars 1988. 